\title{
ON REFLECTION PRINCIPLES FOR PARABOLIC EQUATIONS IN ONE SPACE VARIABLE*
}

\author{
by DAVID COLTON
}

(Received 18th February 1977)

In this note we shall consider the problem of uniquely continuing solutions of the parabolic equation

$$
u_{x x}+a(x, t) u_{x}+b(x, t) u=u_{t}
$$

across an analytic arc $\sigma: x=s_{1}(t)$ on which $u(x, t)$ satisfies the boundary data

$$
\alpha(t) u\left(s_{1}(t), t\right)+\beta(t) u_{x}\left(s_{1}(t), t\right)+\gamma(t) u_{t}\left(s_{1}(t), t\right)=f(t)
$$

We assume that $u(x, t)$ is a classical solution of (1) in the domain $D=$ $\left\{(x, t): s_{1}(t)<x<s_{2}(t), 0<t<t_{0}\right\}$, continuously differentiable in $D \cup \sigma$, and define the "reflection" of $D$ across $\sigma$ by $D^{*}=\left\{(x, t): 2 s_{1}(t)-s_{2}(t)<x<s_{1}(t), 0<t<t_{0}\right\}$. The coefficients $a(x, t)$ and $b(x, t)$ of (1) are assumed to be real analytic in $D \cup \sigma \cup D^{*}$ (although this assumption can be considerably weakened-cf. (1)) and the functions $\alpha(t), \beta(t), \gamma(t)$ and $f(t)$ are assumed to be real analytic on $\left(0, t_{0}\right)$. In (3) (see also (1)) the following reflection principle was proved:

Theorem I. Let $u(x, t)$ be a classical solution of (1) in $D$, continuously different $i$ able in $D \cup \sigma$, and satisfying (2) for $s_{1}(t)=0, \gamma(t)=0$, and $\beta(t) \neq 0$ for $t \in\left(0, t_{0}\right)$. If either

a) $2 \alpha(t)-\beta(t) a(0, t) \neq 0 \quad$ for $t \in\left(0, t_{0}\right)$, or

b) $2 \alpha(t)-\beta(t) a(0, t) \equiv 0$ for $t \in\left(0, t_{0}\right)$,

then $u(x, t)$ can be uniquely continued as a solution of (1) into $D \cup \sigma \cup D^{*}$.

As was pointed out in (3), this theorem is unsatisfactory in the sense that it appears that the continuation property depends on an algebraic relation between the coefficients of the differential equation and the boundary condition. This defect becomes even more pronounced if we consider more general domains where $s_{1}(t) \neq 0$, since if in such cases we make the change of variables

$$
\begin{gathered}
\xi=x-s_{1}(t) \\
\tau=t
\end{gathered}
$$

transforming $x=s_{1}(t)$ into $\xi=0$, it appears that the possibility of continuation

\footnotetext{
* This research was supported in part by AFOSR Grant 76-2879.
} 
depends on a relationship between the coefficients of the differential equation and boundary condition as well as the arc $x=s_{1}(t)$. In this note we remove these difficulties, as well as the assumption that $\gamma(t)=0$, and prove the following result:

Theorem II. Let $u(x, t)$ be a classical solution of (1) in $D$, continuously differentiable in $D \cup \sigma$, and satisfying (2) on $\sigma$. If, for $t \in\left(0, t_{0}\right)$, the nonzero vector $\vec{\mu}(t)=$ $(\beta(t), \gamma(t))$ is never tangent to $x=s_{1}(t)$ (or always tangent to $x=s_{1}(t)$ ) and never parallel to the $x$-axis, (or always parallel to the $x$-axis), then $u(x, t)$ can be uniquely continued as a solution of (1) into $D \cup \sigma \cup D^{*}$.

Proof of Theorem II. The transformation (3) changes (1) into an equation of the same form and the boundary data (2) into the same form with $x=s_{1}(t)$ replaced by $x=0$ and $\beta(t)$ replaced by $\beta(t)-\gamma(t) \dot{s}_{1}(t)$. Noting that the normal to $x=s_{1}(t)$ is given by $\left(1,-\dot{s}_{1}(t)\right)$ we see that $\beta(t)-\gamma(t) \dot{s}_{1}(t)=0$ if and only if $(\beta(t), \gamma(t))$ is tangent to the curve $x=s_{1}(t)$. Hence without loss of generality we can consider (1), (2) with $s_{1}(t)=0$ and assume that $\beta(t) \equiv 0$ or $\beta(t) \neq 0$ for $t \in\left(0, t_{0}\right)$. By a further change of variables of the form

$$
u(x, t)=v(x, t) \exp \left\{-\frac{1}{2} \int_{0}^{x} a(s, t) d s\right\}
$$

we can assume without loss of generality that in (1) we have $a(x, t)=0$. Finally, by solving an appropriate non-characteristic Cauchy problem for (1) (cf. (3), (1), p. 67) we can assume that $f(t)=0$. Hence we have reduced the general reflection problem (1), (2) to that of continuing solutions of equations of the form

$$
u_{x x}+q(x, t) u=u_{t}
$$

subject to

$$
\alpha(t) u(0, t)+\beta(t) u_{x}(0, t)+\gamma(t) u_{t}(0, t)=0
$$

where $u(x, t)$ is defined in a domain of the form $\tilde{D}=\left\{(x, t): 0<x<s_{2}(t), 0<t<t_{0}\right\}$ and is continuously differentiable in $\tilde{D} \cup \tilde{\sigma}$ where $\tilde{\sigma}: x=0$. The theorem will now be proved by considering the following three special cases:

1. $\beta(t) \equiv 0, \gamma(t) \neq 0$ for $t \in\left(0, t_{0}\right)$.

In this case (6) can be rewritten in the form

$$
u(0, t)=u(0,0) \exp \left\{-\int_{0}^{t} \frac{\alpha(\tau)}{\gamma(\tau)} d \tau\right\}
$$

and the result follows from (1), p. 67

2. $\beta(t) \neq 0$ for $t \in\left(0, t_{0}\right), \quad \gamma(t) \equiv 0$.

This corresponds to the case considered in (3). However we want to avoid conditions of the form a), b) of Theorem $I$. To this end we rewrite (6) in the form

$$
u_{x}(0, t)+\frac{\alpha(t)}{\beta(t)} u(0, t)=0
$$


From (2) we have that

$$
u(x, t)=h(x, t)+\int_{0}^{x} M(s, x, t) h(s, t) d s
$$

will be a solution of (5) in $\tilde{D}$ provided

$$
\begin{gathered}
h_{x x}=h_{t} \text { in } \tilde{D} \\
h_{x}(0, t)=0
\end{gathered}
$$

and

$$
\begin{gathered}
M_{x x}-M_{s s}+q(x, t) M=M_{t} \\
M_{s}(0, x, t)=0 \\
\frac{\partial}{\partial x} M(x, x, t)=-\frac{1}{2} q(x, t) .
\end{gathered}
$$

We now integrate (14) to arrive at the initial condition

$$
M(x, x, t)=-\frac{1}{2} \int_{0}^{x} q(s, t) d s-\frac{\alpha(t)}{\beta(t)}
$$

From the analysis in (2) we can conclude that $M(s, x, t)$ exists in $\tilde{D} \cup \tilde{\sigma} \cup \tilde{D}^{*}$ where $\tilde{D}^{*}$ denotes the reflection of $\tilde{D}$ across $\tilde{\sigma}$, and from (9), (10), (15) we have

$$
\begin{aligned}
u_{x}(0, t) & =h_{x}(0, t)+M(0,0, t) h(0, t) \\
& =-\frac{\alpha(t)}{\beta(t)} u(0, t)
\end{aligned}
$$

i.e. $u(x, t)$ satisfies (8). Conversely it follows from an analysis similar to that in (2) that every solution of (5), (8) in $\tilde{D}$ that is continuously differentiable in $\tilde{D} \cup \tilde{\sigma}$ can be represented in the form (9). Since $h(x, t)$ is a solution of the heat equation satisfying $h_{x}(0, t)=0$, the reflection principle for (5), (8) follows from the reflection principle for the heat equation (5). The uniqueness of the continuation follows from Holmgren's uniqueness theorem (cf. 4).

3. $\beta(t) \neq 0$ and $\gamma(t) \neq 0$ for $t \in\left(0, t_{0}\right)$.

If in (5), (6) we set

$$
u(x, t)=v(x, t) \exp \left\{-\int_{0}^{t} \alpha(\tau) d \tau\right\}
$$

we arrive at equations of the form (5), (6) with $\alpha(t)=0$. Hence without loss of generality we consider (5), (6) with $\alpha(t)=0$, i.e. (6) becomes

$$
u_{x}(0, t)+\eta(t) u_{t}(0, \tau)=0
$$

where $\eta(t)=(\gamma(t) / \beta(t))$. Let $h^{(1)}(x, t)$ be a solution of $h_{x x}^{(1)}=h_{t}^{(1)}$ in $\bar{D}$ such that $h_{x}^{(1)}(0, t)=0$, and define $h^{(2)}(x, t)$ by

$$
h^{(2)}(x, t)=-\eta(t) h_{x}^{(1)}(x, t)
$$


Then $h^{(2)}(x, t)$ satisfies

$$
h_{x x}^{(2)}+\frac{\eta^{\prime}(t)}{\eta(t)} h^{(2)}=h_{t}^{(2)}
$$

Note that by the reflection principle for the heat equation $h^{(1)}(x, t)$ and $h^{(2)}(x, t)$ are in fact classical solutions of $(10)$ and (20) respectively in $\bar{D} \cup \tilde{\sigma} \cup \bar{D}^{*}$. We now look for a solution of (5), (18) in the form

$$
\begin{aligned}
u(x, t)= & h^{(1)}(x, t)+h^{(2)}(x, t)+\int_{0}^{x} K^{(1)}(s, x, t) h^{(1)}(s, t) d s \\
& +\int_{0}^{x} K^{(2)}(s, x, t) h^{(2)}(s, t) d s
\end{aligned}
$$

From (2) and (3) it is seen that $u(x, t)$ as defined by (21) will be a solution of (5) provided

$$
\begin{gathered}
K_{x x}^{(1)}-K_{s s}^{(1)}+q(x, t) K^{(1)}=K_{t}^{(1)} \\
K^{(1)}(x, x, t)=-\frac{1}{2} \int_{0}^{x} q(s, t) d s \\
K_{s}^{(1)}(0, x, t)=0
\end{gathered}
$$

and

$$
\begin{gathered}
K_{x x}^{(2)}-K_{s s}^{(2)}+\left[q(x, t)-\frac{\eta^{\prime}(t)}{\eta(t)}\right] K^{(2)}=K_{t}^{(2)} \\
K^{(2)}(x, x, t)=-\frac{1}{2} \int_{0}^{x}\left[q(s, t)-\frac{\eta^{\prime}(t)}{\eta(t)}\right] d s \\
K^{(2)}(0, x, t)=0 .
\end{gathered}
$$

The existence of the kernels $K^{(1)}(s, x, t)$ and $K^{(2)}(s, x, t)$ in $\tilde{D} \cup \tilde{\sigma} \cup \tilde{D}^{*}$ follows from the results of (2). From (21) we have

$$
\begin{aligned}
u_{x}(0, t)+\eta(t) u_{t}(0, t) & =h_{x}^{(1)}(0, t)+h_{x}^{(2)}(0, t)+\eta(t)\left[h_{t}^{(1)}(0, t)+h_{t}^{(2)}(0, t)\right] \\
& =\eta(t)\left[h_{t}^{(1)}(0, t)-h_{x x}^{(1)}(0, t)\right] \\
& =0
\end{aligned}
$$

i.e. $u(x, t)$ satisfies the boundary data (18). The fact that every solution $u(x, t)$ of $(5)$, (18) can be represented in the form (21) follows from an argument similar to that used in (3). The reflection principle for (5), (18) now follows from (21) and the fact that $h^{(1)}(x, t)$ (and hence $\left.h^{(2)}(x, t)\right)$ can be continued into $\tilde{D} \cup \tilde{\sigma} \cup \tilde{D}^{*}$ by the reflection principle for the heat equation. The uniqueness of the continuation again follows from Holmgren's theorem.

Acknowledgement. The fact that the assumption $\gamma(t)=0$ could be removed from Theorem I was first suggested to me by Professor William Rundell. 


\section{REFERENCES}

(1) D. Colton, Solution of Boundary Value Problems by the Method of Integral Operators (Pitman Press, London, 1976).

(2) D. COLTON, Integral operators and reflection principles for parabolic equations in one space variable, J. Diff. Eqns. 15 (1974), 551-559.

(3) D. Colton, Generalized reflection principles for parabolic equations in one space variable, Duke Math. J. 41 (1974), 547-553.

(4) P. Garabedian, Partial Differential Equations (John Wiley, New York, 1964).

(5) D. WIDDER, The Heat Equation (Academic Press, New York, 1975).

UNIVERSITY OF STRATHCLYDE

GLASGOW 